\title{
Correlation between Serum 25-Hydroxyvitamin D3 and Abnormal Insulin Secretion in Patients with Type 2 Diabetes Mellitus
}

\author{
YANI HOU, XIAOJIA HU, JI CHEN ${ }^{1 *}$ AND YUHU CHENG ${ }^{2}$ \\ School of Medicine, Xi'an Peihua University, Xi'an 710125, Shannxi Province, ${ }^{1}$ Department of Endocrinology, Zhuji People's \\ Hospital of Zhejiang Province, Zhuji 311800, Zhejiang Province, ${ }^{2}$ No. 1 Clinical Medical College of Shanxi Datong Universi- \\ ty, Datong No. 5 People's Hospital, Datong 037009, Shanxi Province, Medical College of Shanxi Datong University, Datong \\ 037009, Shanxi Province, China
}

Hou et al.: 25-Hydroxyvitamin D3 and Type 2 Diabetes Mellitus

To investigate the correlation between serum 25-hydroxyvitamin D3 and abnormal insulin secretion in patients with type 2 diabetes mellitus. Eight type 2 diabetes mellitus patients treated from January 2018 to November 2020 (observation group) and another 80 healthy volunteers receiving physical examination in the same period (control group) were included. Fasting venous blood sample was collected to detect serum 25-hydroxyvitamin D3, fasting blood glucose, glycosylated hemoglobin, fasting insulin, insulin resistance index and insulin secretion index. According to insulin secretion index, observation group was divided into normal and abnormal insulin secretion groups and their serum 25-hydroxyvitamin D3, fasting blood glucose, glycosylated hemoglobin, fasting insulin and insulin resistance index were compared. Based on serum 25-hydroxyvitamin D3 level, observation group was divided into serum 25-hydroxyvitamin D3 positive and negative groups and their fasting blood glucose, glycosylated hemoglobin, fasting insulin, insulin resistance index and insulin secretion index were compared. The correlations of serum 25-hydroxyvitamin D3 with fasting blood glucose, glycosylated hemoglobin, fasting insulin, insulin resistance index and insulin secretion index were analyzed. Observation group had lower serum 25-hydroxyvitamin D3 level and insulin secretion index and higher fasting blood glucose, glycosylated hemoglobin, fasting insulin and 
insulin resistance index than those of control group $(p<0.05)$. Abnormal insulin secretion group had lower serum 25-hydroxyvitamin D3 level and higher fasting blood glucose, fasting insulin and insulin resistance index than those of normal insulin secretion group $(\mathrm{p}<0.05)$. Serum 25 -hydroxyvitamin $\mathrm{D} 3$ positive group had higher fasting blood glucose, glycosylated hemoglobin, fasting insulin and insulin resistance index and lower insulin secretion index than those of serum 25-hydroxyvitamin D3 negative group $(p<0.05)$. Serum 25-hydroxyvitamin D3 was negatively correlated with fasting blood glucose, glycosylated hemoglobin, fasting insulin and insulin resistance index, but positively correlated with insulin secretion index. In type 2 diabetes mellitus patients, the expression of serum 25-hydroxyvitamin D3 is down-regulated, which is closely related to abnormal insulin secretion. Serum 25-hydroxyvitamin D3 can be used to determine the insulin secretion function of type 2 diabetes mellitus patients.

Key words: Type 2 diabetes mellitus, insulin secretion, 25-hydroxyvitamin D3, correlation

Type 2 diabetes mellitus (T2DM), a common chronic metabolic disease, is mainly characterized by insulin resistance (IR) and it easily leads to a variety of complications, seriously harming the physical health of patients ${ }^{[1-3]}$. In clinical practice, it is advocated to diagnose and treat T2DM as early as possible. However, in recent years, increasing studies have verified that 25-hydroxyvitamin D3 [25-(OH)D3] plays a vital role in the occurrence and development of $\mathrm{T}_{2} \mathrm{DM}^{[4]}$. In this study, 80 T2DM patients treated in our hospital from January 2018 to November 2020 were compared with another 80 healthy volunteers to determine the correlation between serum 25-(OH)D3 and abnormal insulin secretion in T2DM patients, aiming to investigate the expression and clinical significance of serum 25-(OH)D3 in T2DM. Eight T2DM patients treated from January 2018 to November 2020 (observation group) and another 80 healthy volunteers receiving physical examination in the same period (control group) were included. In control group, there were 43 males and 37 females' aged 50-84 y old and (65.43 \pm 9.14$)$ y old on average. In observation group, there were 44 males and 36 females' aged 50-85 y old and (65.68 \pm 9.32$)$ y old on average. There were no significant differences in the age and gender between the two groups ( $>>0.05)$. This study was approved by the Ethics Committee of our hospital and all patients signed the informed consent. Inclusion criteria: Patients meeting the diagnostic criteria in the Chinese Guidelines for the Prevention and Treatment of T2DM (2017 Edition) ${ }^{[5]}$, confirmed by symptom observation and blood glucose test; those aged $\geq 50$ y old; those who were conscious and would cooperate in the study. Exclusion criteria: Patients with consciousness disorder or mental disorder; those with severe infection, malignant tumor or hematological disease; or those with other chronic underlying diseases.

*Address for correspondence E-mail: chenji20200305@163.com
On the day of examination, the early morning fasting venous blood $(5 \mathrm{~mL})$ was collected from each subject as blood test sample and stored in a special vacuum anticoagulant tube for blood test. After the blood sample was centrifuged at $3000 \mathrm{rpm}$ and $10 \mathrm{~cm}$ radius for $10 \mathrm{~min}$ continuously, the serum was collected. Later, the serum 25-(OH)D3 level was determined by high-performance liquid chromatography-tandem mass spectrometry, fasting blood glucose (FBG) was measured by glucose oxidase method, glycosylated hemoglobin (HbAlc) was detected by highperformance liquid chromatography and fasting insulin (FI) was determined by immunochromatography. IR index and insulin secretion index were calculated as follows: IR index $=\mathrm{FI} \times \mathrm{FBG} / 22.5$, insulin secretion index $=20 \times \mathrm{FI} /(\mathrm{FBG}-3.5)$. Serum 25-(OH)D3, FBG, $\mathrm{HbAlc}$, FI, IR index and insulin secretion index were compared between observation group and control group. According to insulin secretion index, patients in observation group were divided into normal insulin secretion group (insulin secretion index $\geq 50$ ) and abnormal insulin secretion group (insulin secretion index <50) and serum 25-(OH)D3, FBG, HbAlc, FI and IR index were compared between the two groups. Based on the serum 25-(OH)D3 level, patients in observation group were divided into serum $25-(\mathrm{OH})$ D3 positive group $(<7.37 \mu \mathrm{g} / \mathrm{L})$ and serum $25-(\mathrm{OH})$ D3 negative group $(\geq 7.37 \mu \mathrm{g} / \mathrm{L})$ and $\mathrm{FBG}, \mathrm{HbAlc}$, FI, IR index and insulin secretion index were compared between the two groups. Moreover, the correlations

This is an open access article distributed under the terms of the Creative Commons Attribution-NonCommercial-ShareAlike 3.0 License, which allows others to remix, tweak, and build upon the work non-commercially, as long as the author is credited and the new creations are licensed under the identical terms

Accepted 27 February 2021 Revised 04 December 2020 Received 12 August 2020 Indian J Pharm Sci 2021;83(1):158-161 
of serum 25-(OH)D3 with FBG, HbAlc, FI, IR index and insulin secretion index were analyzed in T2DM patients. SPSS 26.0 software was utilized for statistical analysis. The $\chi^{2}$ test was performed for numerical data (n) and the t-test was used to examine the measurement data $(\overline{\mathrm{x}} \pm \mathrm{s}) . \mathrm{p}<0.05$ suggested that a difference was statistically significant. Spearman's test was employed to analyze the correlations and $\mathrm{p}<0.05$ suggested a linear correlation. $r>0$ indicated a positive correlation and $r<0$ implied a negative correlation. Observation group had lower serum 25-(OH)D3 level and insulin secretion index and higher FBG, HbAlc, FI and IR index than those of control group $(p<0.05)$ (Table 1). Among the 80 T2DM patients, 59 had abnormal insulin secretion and 21 had normal insulin secretion. Abnormal insulin secretion group had a lower serum 25-(OH)D3 level and higher FBG, HbAlc, FI and IR index than normal insulin secretion group $(\mathrm{p}<0.05)$ (Table 2). Among the 80 T2DM patients, 54 were positive for serum 25$(\mathrm{OH}) \mathrm{D} 3$ and 26 were negative for serum 25-(OH)D3. Serum 25-(OH)D3 positive group had higher FBG, HbAlc, FI and IR index and lower insulin secretion index than serum $25-(\mathrm{OH}) \mathrm{D} 3$ negative group $(\mathrm{p}<0.05)$ (Table 3). Serum 25-(OH)D3 was negatively correlated with FBG, HbAlc, FI and IR index, but positively correlated with insulin secretion index in T2DM patients (Table 4). T2DM, a chronic disease with a long disease course, is clinically manifested as abnormal increase of blood glucose level. Patients with T2DM are often accompanied by symptoms such as thirst, polyuria and fatigue, seriously affecting their physical and mental health $^{[6-8]}$. T2DM is more common in the elderly patients. In recent years, with the increase in the elderly population, the incidence rate of T2DM has shown an upward trend, and this disease has become a public health problem endangering people's health ${ }^{[9]}$. The pathogenesis of T2DM has not been fully elucidated and there is still a lack of means to cure T2DM, so the major treatment goal is to control blood glucose ${ }^{[10]}$. Insulin is the major means of clinical treatment of T2DM and it suppresses the secretion of insulin in the body mainly through exogenous supplement of insulin, so as to reduce insulin and IR in the body, thereby controlling the blood glucose level ${ }^{[11]}$. Prior to treatment of T2DM, it is necessary to clarify the condition of T2DM patients and whether they have abnormal insulin secretion. Vitamin D is the main substance which regulates calcium and phosphorus metabolism in the body clinically. After entering the human blood, vitamin D can be converted into $25-(\mathrm{OH}) \mathrm{D} 3$ by 25 -hydroxylase in the liver and stored in the human body in the form of $25-(\mathrm{OH}) \mathrm{D} 3$, which is then converted into active 1, $25-(\mathrm{OH}) \mathrm{D} 3$ by 1 -hydroxylase in the kidney. After binding to pancreatic vitamin D receptor, 1,25-(OH)D3 can effectively activate calcium channels in human $\beta$ cells and release insulin, promoting tyrosine phosphorylation of insulin receptor substrate, stimulating insulin signal transduction and thus improving insulin secretion ${ }^{[12,13]}$. In recent years, there have been increasing reports on the correlation between $25-(\mathrm{OH}) \mathrm{D} 3$ and T2DM. In this study, the association between 25-(OH)D3 and T2DM was investigated and it was found that: Observation

TABLE 1: DETECTION INDICES OF OBSERVATION AND CONTROL GROUPS ( $\overline{\mathbf{x}} \pm \mathbf{s})$

\begin{tabular}{lcccccc}
\hline Group & $25-(\mathrm{OH}) \mathrm{D} 3(\mu \mathrm{g} / \mathrm{L})$ & FBG $(\mathrm{mmol} / \mathrm{L})$ & HbAlc $(\%)$ & $\mathrm{FI}(\mathrm{mU} / \mathrm{L})$ & \multicolumn{1}{c}{ IR index } & Insulin secretion index \\
\hline Observation $(\mathrm{n}=80)$ & $6.57 \pm 2.14$ & $8.69 \pm 1.58$ & $7.36 \pm 1.89$ & $30.41 \pm 9.94$ & $3.05 \pm 0.76$ & $46.25 \pm 9.36$ \\
Control $(\mathrm{n}=80)$ & $15.73 \pm 4.85$ & $5.02 \pm 1.07$ & $5.19 \pm 1.30$ & $18.56 \pm 5.27$ & $2.30 \pm 0.61$ & $59.74 \pm 12.08$ \\
$\mathrm{t}$ & 15.455 & 17.202 & 8.461 & 9.421 & 6.884 & 7.896 \\
$\mathrm{p}$ & 0.000 & 0.000 & 0.000 & 0.000 & 0.000 & 0.000 \\
\hline
\end{tabular}

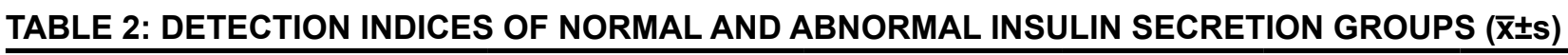

\begin{tabular}{lccccc}
\hline Group & $25-(\mathrm{OH}) \mathrm{D} 3(\boldsymbol{\mu g} / \mathrm{L})$ & FBG $(\mathrm{mmol} / \mathrm{L})$ & HbAlc $(\%)$ & $\mathrm{FI}(\mathrm{mU} / \mathrm{L})$ & IR index \\
\hline Abnormal insulin secretion $(\mathrm{n}=59)$ & $5.95 \pm 1.03$ & $9.23 \pm 0.98$ & $7.89 \pm 1.02$ & $32.97 \pm 4.76$ & $3.24 \pm 0.39$ \\
Normal insulin secretion $(\mathrm{n}=21)$ & $7.18 \pm 1.20$ & $8.14 \pm 0.87$ & $6.81 \pm 0.94$ & $28.03 \pm 4.15$ & $2.85 \pm 0.36$ \\
$\mathrm{t}$ & 4.498 & 4.501 & 4.250 & 4.216 & 4.012 \\
$\mathrm{p}$ & 0.000 & 0.000 & 0.000 & 0.000 & 0.000 \\
\hline
\end{tabular}

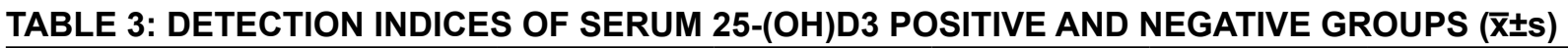

\begin{tabular}{lccccc}
\hline Group & FBG $(\mathrm{mmol} / \mathrm{L})$ & HbAlc $(\%)$ & $\mathrm{FI}(\mathrm{mU} / \mathrm{L})$ & IR index & Insulin secretion index \\
\hline Serum 25-(OH)D3 positive $(\mathrm{n}=54)$ & $9.16 \pm 0.95$ & $7.78 \pm 0.89$ & $32.73 \pm 4.20$ & $3.21 \pm 0.34$ & $44.37 \pm 3.18$ \\
Serum 25-(OH)D3 negative $(\mathrm{n}=26)$ & $8.21 \pm 0.84$ & $6.90 \pm 0.75$ & $28.25 \pm 3.51$ & $2.87 \pm 0.31$ & $48.09 \pm 3.60$ \\
$\mathrm{t}$ & 4.344 & 4.349 & 4.702 & 4.307 & 4.693 \\
$\mathrm{p}$ & 0.000 & 0.000 & 0.000 & 0.000 & 0.000 \\
\hline
\end{tabular}


TABLE 4: CORRELATION ANALYSIS RESULTS

\begin{tabular}{lcc}
\hline \multirow{2}{*}{ Index } & \multicolumn{2}{c}{$25-(\mathrm{OH}) \mathrm{D} 3$} \\
\cline { 2 - 3 } FBG & $\mathrm{r}$ & $\mathrm{p}$ \\
HbAlc & -0.790 & 0.006 \\
$\mathrm{FI}$ & -0.802 & 0.005 \\
IR index & 0.879 & 0.002 \\
Insulin secretion index & -0.797 & 0.006 \\
\hline
\end{tabular}

group had lower serum 25-(OH)D3 level and insulin secretion index and higher FBG, HbAlc, FI and IR index than control group $(\mathrm{p}<0.05)$; Abnormal insulin secretion group had a lower serum 25-(OH)D3 level and higher FBG, FI and IR index than normal insulin secretion group $(\mathrm{p}<0.05)$; Serum $25-(\mathrm{OH}) \mathrm{D} 3$ positive group had higher FBG, HbAlc, FI and IR index and lower insulin secretion index than serum 25-(OH) D3 negative group $(\mathrm{p}<0.05)$; Serum $25-(\mathrm{OH}) \mathrm{D} 3$ was negatively correlated with FBG, HbAlc, FI and IR index, but positively correlated with insulin secretion index in T2DM patients. The above results suggest that there is a positive correlation between serum $25-(\mathrm{OH})$ D3 and insulin secretion index in T2DM patients. The down regulated serum 25-(OH)D3 level indicates that the insulin secretion may be abnormal and IR may occur in T2DM patients, so it is necessary to monitor their blood glucose level so as to strengthen blood glucose control. In summary, the expression of serum $25-(\mathrm{OH})$ D3 is generally down regulated in T2DM patients, which is closely related to abnormal insulin secretion. In clinic, serum 25-(OH)D3 can be detected to judge the insulin secretion function of T2DM patients.

\section{Authors' contributions:}

Yuhu Cheng and Xiaofen Guo contributed equally to this study.

\section{Conflicts of interest:}

The authors declared no conflicts of interest.

\section{REFERENCES}

1. Alkhiari R, Alzayer H, Aljazeeri J, Vanniyasingam T, Punthakee $Z$. Adherence to guidelines for inpatient pharmacologic management of type 2 diabetes in adults and glycemic outcomes. Can J Diabetes 2018;42(2):158-62.

2. Vajda EG, Logan D, Lasseter K, Armas D, Plotkin DJ, Pipkin $\mathrm{JD}$, et al. Pharmacokinetics and pharmacodynamics of single and multiple doses of the glucagon receptor antagonist LGD6972 in healthy subjects and subjects with type 2 diabetes mellitus. Diabetes Obes Metab 2017;19(1):24-32.

3. Zhu B, Hershberger PE, Kapella MC, Fritschi C. The relationship between sleep disturbance and glycaemic control in adults with type 2 diabetes: an integrative review. J Clin Nurs 2017;26(23-24):4053-64.

4. Kocot J, Dziemidok P, Kiełczykowska M, Kurzepa J, Szcześniak G, Musik I. Is There Any Relationship between Plasma 25-Hydroxyvitamin D3, Adipokine Profiles and Excessive Body Weight in Type 2 Diabetic Patients? Int J Environ Res Public Health 2018;15(1):19.

5. Chinese Diabetes Society. Chin J Pract Internal Med 2018;10(1):292-344.

6. Riek AE, Oh J, Darwech I, Worthy V, Lin X, Ostlund Jr RE, et al. Vitamin D3 supplementation decreases a unique circulating monocyte cholesterol pool in patients with type 2 diabetes. J Steroid Biochem Mol Biol 2018;177:187-92.

7. Wang W, Ye S, Qian L, Xing X. Sex-specific association of serum 25-hydroxyvitamin D3 with insulin resistance in Chinese Han patients with newly diagnosed type 2 diabetes mellitus. J Nutr Sci Vitaminol 2018;64(3):173-8.

8. Samefors M, Scragg R, Länne T, Nyström FH, Östgren CJ. Association between serum $25(\mathrm{OH}) \mathrm{D} 3$ and cardiovascular morbidity and mortality in people with Type 2 diabetes: a community-based cohort study. Diabet Med 2017;34(3):372-9.

9. Ucak S, Sevim E, Ersoy D, Sivritepe R, Basat O, Atay S. Evaluation of the relationship between microalbuminuria and $25-(\mathrm{OH})$ vitamin D levels in patients with type 2 diabetes mellitus. Aging Male 2019;22(2):116-20.

10. Hwang JJ, Jiang L, Hamza M, Rangel ES, Dai F, BelfortDeAguiar R, et al. Blunted rise in brain glucose levels during hyperglycemia in adults with obesity and T2DM. JCI Insight 2017;2(20):e95913.

11. Tangvarasittichai S. Oxidative stress, insulin resistance, dyslipidemia and type 2 diabetes mellitus. World J. Diabetes 2015;6(3):456-80.

12. Mazidi M, Siervo M. The Link between Serum 25-Hydroxyvitamin D, Inflammation and Glucose/Insulin Homeostasis Is Mediated by Adiposity Factors in American Adults. Br J Nutr 2021;1-24.

13. Al-Tu'ma FJ, Yosuf LM. Vitamin D deficiency and hypertension in type 2 diabetic Iraqi patients. J Contemp Med Sci 2015;1(1):17-20. 